\title{
The interstellar turbulent plasma spectrum in the direction to PSR B1642-03 from multi-frequency observations of interstellar scintillation ${ }^{\star}$
}

\author{
T. V. Smirnova ${ }^{1}$, V. I. Shishov ${ }^{1}$, W. Sieber ${ }^{2}$, D. R. Stinebring ${ }^{3}$, V. M. Malofeev ${ }^{1}$, \\ V. A. Potapov ${ }^{1}$, S. A. Tyul'bashev ${ }^{1,4}$, A. Jessner ${ }^{4}$, and R. Wielebinski ${ }^{4}$ \\ 1 Pushchino Radioastronomy Observatory of Lebedev Physical Institute, 142290, Pushchino, Russia, and Isaac Newton Institute \\ of Chile, Pushchino Branch \\ 2 Hochschule Niederrhein, Reinarzstr. 49, 47805 Krefeld, Germany \\ e-mail: Wolfgang.Sieber@hsnr.de \\ 3 Oberlin College, $\mathrm{OH} 44074$, Oberlin, USA \\ 4 Max-Planck-Institut für Radioastronomie, Auf dem Hügel 69, 53121 Bonn, Germany
}

Received 28 May 2004 / Accepted 9 March 2006

ABSTRACT

\begin{abstract}
Multi-frequency observations of interstellar scintillation toward the pulsar PSR B1642-03 were analyzed to estimate the spectrum of interstellar plasma inhomogeneities in the direction of this pulsar. Using data over the frequency range from $103 \mathrm{MHz}$ to $5 \mathrm{GHz}$, we constructed the composite structure function (SF) of phase fluctuations, which covers a corresponding wide range of turbulence scales. The structure function shows that the interstellar plasma spectrum in the direction to this pulsar follows a piecewise power law. The power law is well described by two exponents: $n=3.7$ for scales from $10^{9}$ to $10^{15} \mathrm{~cm}$ (Kolmogorov spectrum) and $n=3.35$ for scales less than $10^{9} \mathrm{~cm}$. We interpret the unusual behaviour of the spectrum to be caused by the line of sight passing through the North Polar Spur, which may have plasma properties similar to the anisotropic plasma of the solar wind, although at a very different density.
\end{abstract}

Key words. stars: pulsars: general - turbulence - ISM: structure - stars: pulsars: individual: PSR B1642-03

\section{Introduction}

It is well known that the analysis of diffractive and refractive scintillation of pulsar signals bears information about the shape of the interstellar plasma spectrum. Such measurements allow the construction of the composite structure function (SF) of phase fluctuations. The SF follows a power law as shown by Armstrong et al. (1995) over a very wide range of scales $\left(10^{6}\right.$ to $\left.10^{13} \mathrm{~m}\right)$ in the nearer interstellar medium (ISM) $(R \leq 1 \mathrm{kpc})$ as well as for greater distances $(R>1 \mathrm{kpc})$ (Shishov \& Smirnova 2001). The data are in a statistical sense well described by a Kolmogorov spectrum. However, the dispersion of the points is large, and the spectrum may differ from a Kolmogorov law in specific directions to given sources. We showed previously (Shishov et al. 2003) that the analysis of multi-frequency observations gives useful information about the ISM in a chosen direction with good accuracy. We found that the spectrum in the direction to PSR B0329+54 is well described by a power law with $n=3.5$ for scales from $10^{6}$ to $10^{9} \mathrm{~m}$, differing from the Kolmogorov value $n=3.67$. It is notable that strong angular refraction was also observed for this line of sight.

In this paper we analyze multi-frequency observations of PSR B1642-03 in the range from $103 \mathrm{MHz}$ to $5 \mathrm{GHz}$. The method of analysis and the basic equations are described in Shishov et al. (2003). We construct the composite structure function of phase fluctuations in the time and frequency domain and

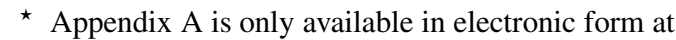
http://www . edpsciences.org compute the spectrum of interstellar plasma inhomogeneities in the direction to this pulsar.

\section{Observations}

\subsection{Diffractive scintillation at $103 \mathrm{MHz}$}

The observations of PSR B1642-03 at 102.6 MHz were carried out in June 1994 with the Large Phased Array (BSA) at Pushchino using a 128-channel receiver with a bandwidth, $\Delta f$, of $1.25 \mathrm{kHz}$ per channel. The 128 channels were sampled every $5.12 \mathrm{~ms}$ in a pulse window of $200 \mathrm{~ms}$ duration synchronized with the pulsar period and were written to disk after detection and summation over eight pulsar periods (3.1 s), which we call a "block" of data. The window included on-pulse and off-pulse contributions. A typical observation lasted for about 70 blocks. The methods of observation are described in more detail by Malofeev et al. (1995).

The signals in adjacent channels were shifted to remove the dispersion delay and scaled so that the variance due to noise had the same amplitude in all channels. We then averaged three bins at the maximum of the mean pulse profile to represent the onpulse intensity of each channel and three bins off the pulse to represent the noise (off-pulse) intensity. On-pulse and off-pulse correlation functions could then be evaluated from these data. In keeping with convention (Cordes et al. 1985), the characteristic frequency and time scales $\Delta f_{\mathrm{d}}$ and $\Delta t_{\mathrm{d}}$ were defined as half of the autocorrelation function (ACF) width at a level of 0.5 along 
Table 1. Frequency and time resolution $\Delta f$ and $\Delta t$ in comparison to the characteristic frequency and time scales $\Delta f_{\mathrm{d}}$ and $\Delta t_{\mathrm{d}}$.

\begin{tabular}{ccccc}
\hline \hline$f, \mathrm{MHz}$ & $\Delta f, \mathrm{kHz}$ & $\Delta f_{\mathrm{d}}, \mathrm{kHz}$ & $\Delta t, \mathrm{~s}$ & $\Delta t_{\mathrm{d}}, \mathrm{s}$ \\
\hline 102.6 & 1.25 & $0.05 \pm 0.01$ & 3.1 & $6-9$ \\
335 & 10 & $20 \pm 4$ & 10 & $70 \pm 11$ \\
408 & 19.5 & $60 \pm 13$ & 28 & $75 \pm 13$ \\
610 & 19.5 & $570 \pm 160$ & 59 & $130 \pm 30$ \\
800 & 78 & $1730 \pm 570$ & 29 & $170 \pm 46$ \\
4850 & $5 \times 10^{5}$ & - & 15 & $1610 \pm 590$ \\
\hline
\end{tabular}

the frequency axis and at a level of $1 / e$ along the time axis after removing the spike at zero lag due to noise. We also calculated cross-correlation functions (CCF) between spectra of pairs of data blocks (minimum separation $3.1 \mathrm{~s}$ ) separated by 8,16 , and 24 pulses (corresponding to one, two and three blocks).

Significant correlation exists for adjacent blocks (time separation $3.1 \mathrm{~s}$ or eight pulse periods) falling down to the noise level at a separation of three blocks ( 24 pulse periods). The correlation levels are $0.078 \pm 0.011,0.091 \pm 0.028$ and $-0.007 \pm 0.022$ at time separations of $3.1 \mathrm{~s}, 6.2 \mathrm{~s}$ and $9.3 \mathrm{~s}$, respectively. We conclude therefore that $\Delta t_{\mathrm{d}} \cong(6 \div 9) \mathrm{s}$. Numerical simulations of Lee (1975) show that smoothing of the frequency structure by the receiver bandwidth (which is the case for our data; see below) causes a decrease of the modulation index but changes the time scale of intensity fluctuations only weakly.

Our frequency resolution $(\Delta f=1.25 \mathrm{kHz})$ is insufficient to evaluate the decorrelation frequency scale $\Delta f_{\mathrm{d}}$ directly; it is clear only that $\Delta f_{\mathrm{d}} \ll \Delta f$ (see Table 1 ). We may, however, estimate the time scale from the scintillation index $m$ and the value of the cross-correlation coefficient at zero frequency lag $C C F(0)$ :

$m^{2}=C C F(0) \cdot \frac{\left\langle\sigma^{2}\right\rangle}{\left\langle I^{2}\right\rangle}$,

where $\left\langle\sigma^{2}\right\rangle$ and $\left\langle I^{2}\right\rangle$ denote the mean values of the squared variance and intensity calculated from the data for the whole duration of the observations. The measured value averaged over five days of observations is $m^{2}=0.18 \pm 0.03$ or $m=0.42 \pm 0.17$. We would expect $m=1$ if $\Delta f_{\mathrm{d}} \gg \Delta f$ since we are at $102.6 \mathrm{MHz}$ in the regime of strong scintillation. The poor frequency resolution of our receiver smooths the frequency structure and decreases the modulation index. The influence of the receiving bandwidth on the measured scintillation index was numerically evaluated by Lee (1976). His result can be approximated for small ratios of $\Delta f_{\mathrm{d}} / \Delta f$ by the following relation:

$$
\begin{aligned}
m^{2} & =2 A \frac{\Delta f_{\mathrm{d}}}{\Delta f} m_{0}^{2}, \\
A & =\int_{0}^{B / \Delta f_{\mathrm{d}}} \mathrm{d} x C C F(x) \cong 2.1, x=\frac{\delta f}{\Delta f_{\mathrm{d}}},
\end{aligned}
$$

where $B$ is the total bandwidth of the receiver, and $m_{0}$ denotes the modulation index for a receiver with $\Delta f \ll \Delta f_{\mathrm{d}}$; we assume here $m_{0}=1$ (see above). The coefficient $A=2.1$ was calculated using the $\mathrm{CCF}$ at $800 \mathrm{MHz}$ (this $\mathrm{CCF}$ is presented below). Based on these assumption we estimate $\Delta f_{\mathrm{d}}=(0.05 \pm 0.01) \mathrm{kHz}$.

\subsection{Diffractive scintillation at $335 \mathrm{MHz}$ and $408 \mathrm{MHz}$}

At $335 \mathrm{MHz}$, we analyzed the observations of Roberts \& Ables (1982) made with the Parkes 64-m telescope. These observations were made in September 1974 with a 63-channel receiver with a bandwidth of $10 \mathrm{kHz}$ for each channel. Roberts and Ables added
26 pulse periods (10 s) to build one spectrum. The total observing time was 147 min corresponding to 880 successive spectra.

Figures $12 \mathrm{a}$ and $\mathrm{b}$ of their paper show the derived autocovariance functions in frequency and time. They compute after removing the spike at zero lag due to noise - a scintillation time of $\Delta t_{\mathrm{d}}=70 \mathrm{~s}$ and a frequency scale of $\Delta f_{\mathrm{d}}=20 \mathrm{kHz}$. Assuming a frequency scaling law as for a Kolmogorov spectrum of inhomogeneities - that is $f^{4.4}$ and $f^{1.2}$ for $\Delta f_{\mathrm{d}}$ and $\Delta t_{\mathrm{d}}-$ one would get $\Delta f_{\mathrm{d}}=0.11 \mathrm{kHz}$ and $\Delta t_{\mathrm{d}}=17 \mathrm{~s}$ at $102.6 \mathrm{MHz}$.

The data at $408 \mathrm{MHz}$ were taken from the paper of Gupta et al. (1994). They quote a characteristic frequency scale of $\Delta f_{\mathrm{d}}=60 \mathrm{kHz}$ and time scale of $\Delta t_{\mathrm{d}}=75 \mathrm{~s}$. Their frequency and time resolutions were insufficient, however, to compute the frequency and time structure functions in the region of small lags.

\subsection{Diffractive scintillation at $610 \mathrm{MHz}$ and $800 \mathrm{MHz}$}

The observations at $610 \mathrm{MHz}$ and $800 \mathrm{MHz}$ were made with the NRAO 42-m telescope in 1995 and 1992, respectively. We used the 1024 channel NRAO Spectral Processor. At $610 \mathrm{MHz}$, a total bandwidth of $20 \mathrm{MHz}$ in two orthogonal polarizations was used from which the total intensity was computed as the sum of the two signals. The off-pulse spectra were subtracted from the on-pulse spectra. The spectra were accumulated for $59 \mathrm{~s}$ and written to magnetic tape for subsequent off-line analysis. Each observation lasted for about $105 \mathrm{~min}$. At $800 \mathrm{MHz}$, a total bandwidth of $79.8 \mathrm{MHz}$ in one linear polarization was recorded. The spectra were accumulated for $29 \mathrm{~s}$ and the total observing time was 48 min.

The signal-to-noise ratio of the frequency ACFs can be enhanced by averaging over time. We have done this by averaging over $59 \mathrm{~s}$ at $610 \mathrm{MHz}$ (the accumulation time, one block) and over $29 \mathrm{~s}$ at $800 \mathrm{MHz}$. We increased the signal-to-noise ratio of the time ACFs by averaging over the frequency channels, which was done to compute mean time ACFs. In addition, we computed mean cross-correlation functions by cross-correlating the intensity variations in neighbouring time blocks (a separation of either $59 \mathrm{~s}$ or $29 \mathrm{~s}$ ) or frequency channels and then by averaging over all available pairs. The mean frequency and time ACFs and CCFs are presented in Fig. 1 for $f=610 \mathrm{MHz}$ and Fig. 2 for $f=800 \mathrm{MHz}$. All calculated correlation functions were normalized by $\sigma^{2}$ for the ACF or $\sigma_{1} \sigma_{2}$ for the CCF where $\sigma$ is the standard deviation of intensity variations. We did a linear extrapolation of the ACFs near zero time and frequency lags to correct for noise. The frequency resolution at $610 \mathrm{MHz}$ is satisfactory (see Fig. 1), about 20 times narrower than $\Delta f_{\mathrm{d}}$ (see Table 1), but the time resolution is only about twice that of $\Delta t_{\mathrm{d}}$. The frequency and time resolution at $800 \mathrm{MHz}$ is quite good.

It should be remarked that the value of the CCF at $(t, \Delta \tau=0)$ in Fig. 1 is greater than that of the ACF at $(t, \Delta \tau=0)$ because the intensity variations in neighbouring frequency channels are more highly correlated than the variations at the same frequency with a time shift of one lag $\left(\Delta f_{\mathrm{d}} / \Delta_{f} \gg \Delta t_{\mathrm{d}} / \Delta t\right)$ so that the correlation along the time axis is smaller.

We have about 90 independent measurements of $\Delta f_{\mathrm{d}}$ and $\Delta t_{\mathrm{d}}$ at $610 \mathrm{MHz}$ so that their standard deviation with time, $\sigma_{610}$, can be evaluated. The standard deviation amounts to $25 \%$ as shown in Table 1 . These variations are, however, mainly caused by refractive scintillation. The relative variations of the diffractive frequency and time scales are proportional to the modulation index of refractive scintillation (Shishov 2001) so that one may evaluate these variances at other frequencies considering the modulation index for refractive scintillation, $m_{\text {ref }} \propto f^{0.6}$ (Smirnova et al. 1998). In Table 1 error bars are computed for 
PSR B1642-03 $f=610 \mathrm{MHz}$
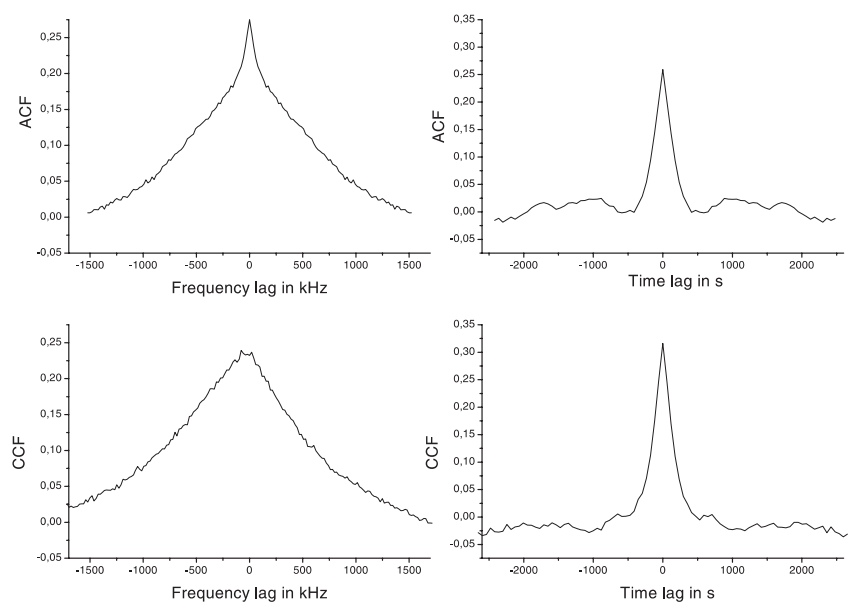

Fig. 1. Left: normalized mean frequency auto- (top) and cross-correlation (bottom) function at $610 \mathrm{MHz}$. One frequency lag corresponds to $19.5 \mathrm{kHz}$. The integration time is $105 \mathrm{~min}$ and the time shift for cross-correlated spectra amounts to $59 \mathrm{~s}$. Right: mean time auto- (top) and cross-correlation (bottom) function at $610 \mathrm{MHz}$. One time lag corresponds to $59 \mathrm{~s}$. The integration covers 1024 frequency channels and the frequency shift for the cross-correlation is $19.5 \mathrm{kHz}$.

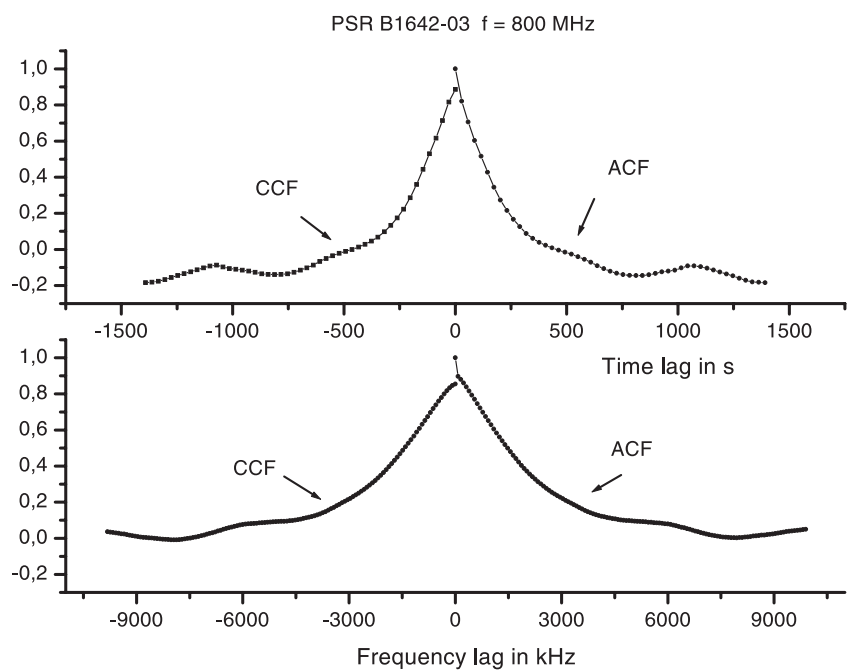

Fig. 2. Bottom: mean frequency auto-correlation function (right) combined with the cross-correlation function (left) between successive spectra at $800 \mathrm{MHz}$. One frequency lag corresponds to $77.9 \mathrm{kHz}$. The integration time is $48 \mathrm{~min}$. Top: mean time auto-correlation function (right) combined with the cross-correlation function (left). One time lag corresponds to $29 \mathrm{~s}$. The integration covers 1024 frequency channels and the frequency shift for the cross-correlation is $77.9 \mathrm{kHz}$.

the frequencies $335 \mathrm{MHz}, 308 \mathrm{MHz}$, and $800 \mathrm{MHz}$ according to $\sigma_{f}=\sigma_{610}(f / 610)^{0.6}$. The error at $4850 \mathrm{MHz}$ was estimated by $\sigma=\sqrt{\frac{\Delta t_{\mathrm{d}}}{T}}$ where $T$ is the total observing time.

\subsection{Data at $4.85 \mathrm{GHz}$}

The observations at $4.85 \mathrm{GHz}$ were carried out in July 2001 with the 100-m radio telescope of the Max-Planck-Institut für Radioastronomie. We used a receiver with a bandwidth of $500 \mathrm{MHz}$. The mean system temperature was about $60 \mathrm{~K}$. Individual pulses were sampled 1024 times per pulsar period and integrated subsequently in a data logger over 39 pulsar periods
PSR B1642-03 $f=5 \mathrm{GHz}$
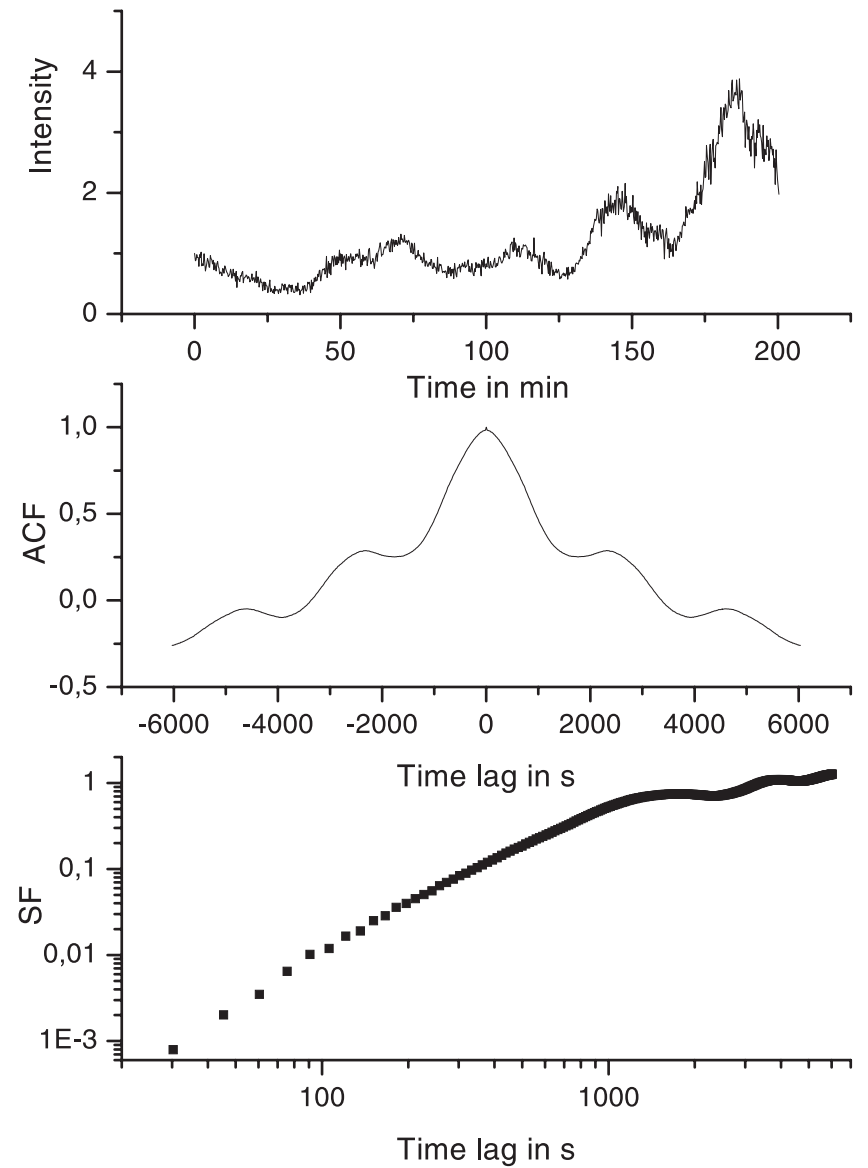

Fig. 3. Top: flux variations (in arbitrary units) at $4.85 \mathrm{GHz}$ covering $200 \mathrm{~min}$. Each data point corresponds to 39 pulsar periods (about $15 \mathrm{~s}$ ). Middle: time auto-correlation function of these variations. Bottom: time structure function. Intensity variations were normalized by the mean value.

(about $15 \mathrm{~s}$ ) to improve the signal-to-noise ratio. Intensity variations for the 200 min observing time are shown in Fig. 3 (top). The corresponding ACF is shown in the middle panel of Fig. 3. The modulation over our still limited observing time shows a quasiperiodic appearance, which might smooth out for longer observations. We used three days of observations for the following analysis and computed a modulation index in the range $0.64 \div 0.82$.

The diffractive parameters as derived from our correlation analysis, as well as the frequency and time resolutions, are presented for all frequencies in Table 1 . Figure 4 presents the dependence of the diffractive parameters $\Delta t_{\mathrm{d}}$ and $\Delta f_{\mathrm{d}}$ on the observing frequency. Linear fits to the log-log scale result in $\Delta t_{\mathrm{d}} \propto f^{\gamma_{1}}$ and $\Delta f_{\mathrm{d}} \propto f^{\gamma_{2}}$ with $\gamma_{1}=1.4 \pm 0.08$ and $\gamma_{2}=5.0 \pm 0.1$. For a power law spectrum of inhomogeneities with slope $n$ one would expect $\gamma_{1}=2 /(n-2)$ and $\gamma_{2}=2 n /(n-2)$, which means $n$ should be near to 3.3 based on the derived values of $\gamma_{1}$ and $\gamma_{2}$. We will show later that this value of $n$ is in good agreement with the results of the structure function analysis.

\section{Results}

Using the equations of Appendix A we have constructed time and frequency structure functions (SFs) based on the derived 


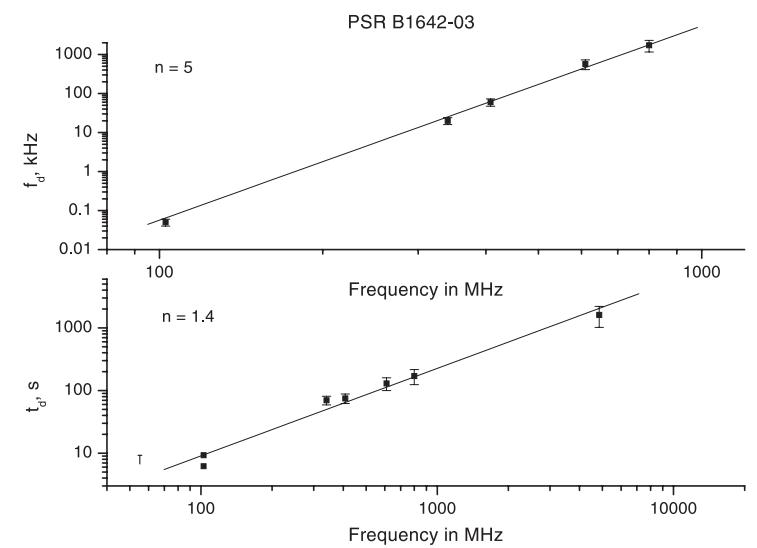

Fig. 4. Frequency dependence of the diffractive scintillation frequency scale (top) and time scale (bottom). The data are taken from Table 1.

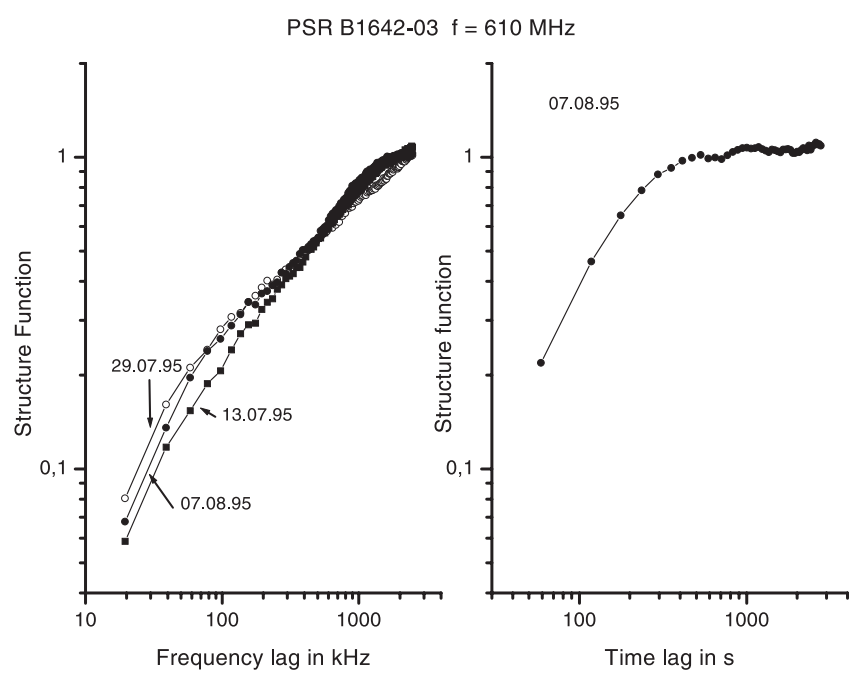

Fig. 5. Time $(r i g h t)$ and frequency $(l e f t)$ intensity structure function at $610 \mathrm{MHz}$ calculated for different days of observation. Structure functions were normalized by the intensity variance squared.

ACFs which were corrected for noise and reduced to the frequency $f_{0}=1000 \mathrm{MHz}$. The structure functions can be modified by two factors in addition to noise. The first is a modulation of the diffractive pattern by refractive scintillation. This is demonstrated in Fig. 5, where examples of frequency structure functions are shown at $610 \mathrm{MHz}$ calculated for different days of observation. The shape of the structure functions varies clearly for small frequency lags. The variation is mainly caused by different values of the flux variance used for normalization. The SFs are very similar for small frequency lags without this normalization.

The second factor that may modify a SF is given by the smoothing of the frequency structure by integration over time. Integration along the time axis leads to a suppression of smallscale structure along the frequency axis and to a distortion of the frequency structure function at small values of frequency lags. Similarly, integration along the frequency axis (or insufficient frequency resolution) can lead to a distortion of the time structure function. Our measured frequency structure functions show, as a consequence of this mutual interdependence, a steepening at small frequency lags (Fig. 5). This steepening is caused by poor time resolution. The lack of short-time measurements causes the time structure function to stop at a level of 0.2 (Fig. 5 right), which influences the frequency structure function at these low
PSR B1642-03
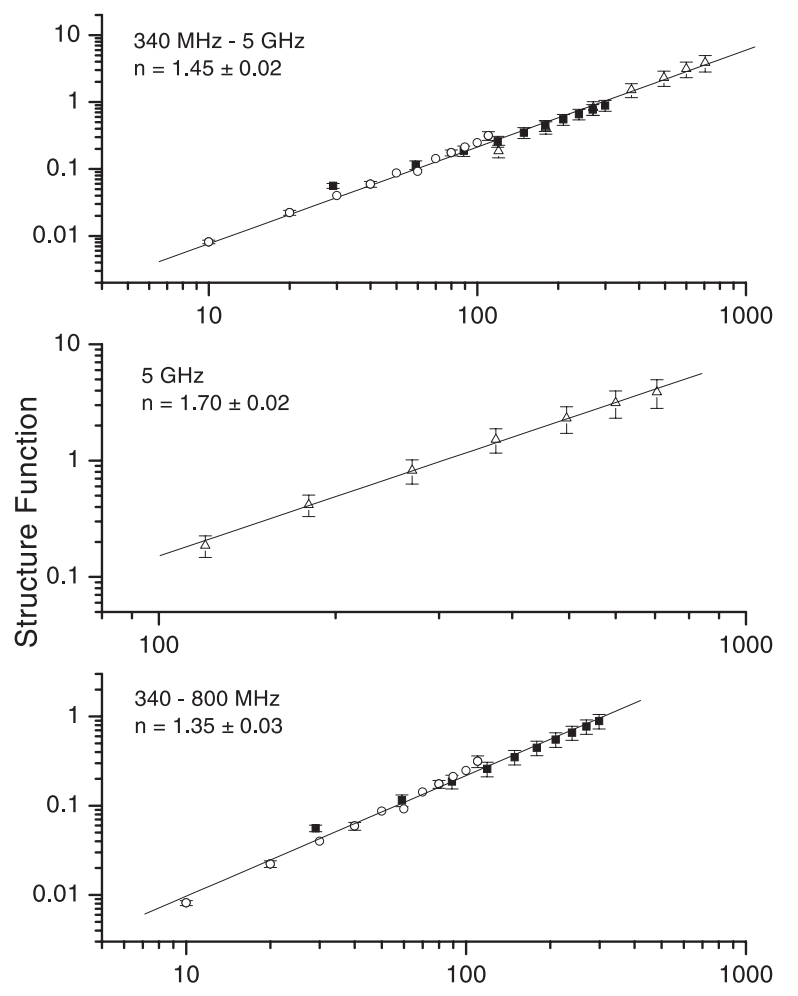

Time lag in s

Fig. 6. Time structure function of phase fluctuations (in radians ${ }^{2}$ ) reduced to $f_{0}=1 \mathrm{GHz}$ as compiled from data based on different frequency ranges: $(340 \div 800) \mathrm{MHz}($ bottom), $4.85 \mathrm{GHz}$ (middle) and $340 \mathrm{MHz} \div 4.85 \mathrm{GHz}$. Data points are marked with the following symbols: $340 \mathrm{MHz}$ - open circles; the mean SF based on data at $610 \mathrm{MHz}$ and $800 \mathrm{MHz}$ - filled squares; $4.85 \mathrm{GHz}$ - triangles. The solid lines correspond to the best fits to the corresponding data points.

levels. The minimum levels of the time and frequency structure functions should therefore be consistent with each other.

Figure 6 shows the time structure functions $D_{\mathrm{S}}(t)$ in a log-log diagram calculated for different frequency ranges converted to one reference frequency, $1000 \mathrm{MHz}$ (Eq. (A.9)). The frequency ranges are: $(340 \div 800) \mathrm{MHz}, 4.85 \mathrm{GHz}$, and $340 \mathrm{MHz} \div 4.85 \mathrm{GHz}$. We used the exact relation (Eq. (A.4)) to compute the $\mathrm{SF}$ in the range from $340 \mathrm{MHz}$ to $800 \mathrm{MHz}$ and Eq. (A.3) for $4.85 \mathrm{GHz}$. The main effect that causes scatter in the calculated SF at different observation epochs is the modulation of the diffractive pattern by refractive scintillation. It is well known that the characteristic time and frequency scales of diffractive scintillation can change in time by up to a factor of 2 to 3 . We averaged the structure functions of three days at $610 \mathrm{MHz}$ and one day at $800 \mathrm{MHz}$ to improve the statistics. To use the same time resolution (29s) at $610 \mathrm{MHz}$ as for $800 \mathrm{MHz}$ we interpolated the corresponding SFs before averaging. The estimation errors for structure functions were defined using equation (B12) from the paper of Rickett et al. (2000) taking into account that all SFs were reduced to $f_{0}=1 \mathrm{GHz}$. We mention that the error of the $\operatorname{SF}\left(\tau=\Delta t_{\mathrm{d}}\right)$ at $340 \mathrm{MHz}$ is about a factor of two greater than the statistical error at this scale.

The structure function at $4.85 \mathrm{GHz}$ was calculated by averaging the SFs from 3 days of observation and using time lags from $120 \mathrm{~s}$ to $0.7 \Delta t_{\mathrm{d}}$ (to exclude the steepening caused by insufficient frequency resolution). The data points at different 


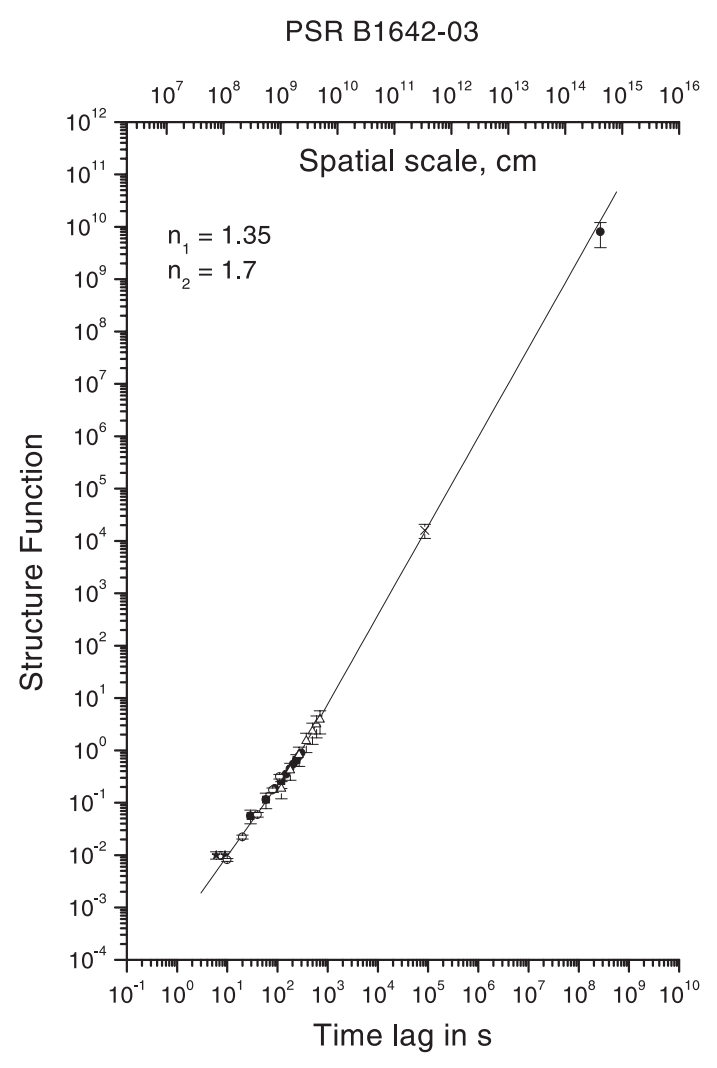

Fig. 7. Time structure function of phase fluctuations (in radians ${ }^{2}$ ) reduced to $f_{0}=1 \mathrm{GHz}$ as compiled from the following data points: 1) $102 \mathrm{MHz}$ observations - stars; 2) $340 \mathrm{MHz}$ - open circles; 3) $800 \mathrm{MHz}$ - filled squares; 4) $4.85 \mathrm{GHz}$ - triangles; 5) refractive scintillation at $610 \mathrm{MHz}$ - cross; 6) timing at $103 \mathrm{MHz}$ - filled circle. The two solid lines correspond to fits to the structure function with a slope of 1.35 in the range $(3 \div 200)$ s and with a slope of 1.7 for time lags larger than $200 \mathrm{~s}$.

frequencies are marked by different symbols. The fits, corresponding to different frequency ranges, are shown in Fig. 6 by solid lines. The structure functions based on the $340 \mathrm{MHz}$ to $800 \mathrm{MHz}$ and $4.85 \mathrm{GHz}$ data points have different slopes: $\alpha=1.35 \pm 0.03$ and $\alpha=1.70 \pm 0.02$, respectively. The fit over the whole range from $340 \mathrm{MHz}$ to $4.85 \mathrm{GHz}$ gives $\alpha=1.45 \pm 0.02$. which differs significantly from the Kolmogorov value of $\alpha=$ 1.67 .

We believe that a piece-wise power law exists for different time scales of scintillation. Figure 7 shows the resulting composite time structure function including data from $102.6 \mathrm{MHz}$ up to $5 \mathrm{GHz}$ and also the evaluation of the SF obtained from refractive scintillation and timing data of PSR B1642-03. We used the refractive scintillation index $\left(m_{\mathrm{ref}}=0.46\right)$ and the refractive time scale $\left(T_{\text {ref }}=0.9\right.$ days $)$ at $610 \mathrm{MHz}$ from Stinebring et al. (2000) to compute a value of the SF at a time lag of about $10^{5} \mathrm{~s}$ according to Eqs. (A.18) and (A.19). For this computation one has to assume a power law index, which we took to be $n=3.67$ (a Kolmogorov spectrum). The error of this estimation of the SF is about $30 \%$, which is based on errors of $m_{\text {ref }}, T_{\text {ref }}$ and $\Delta t_{\mathrm{d}}$. We supplemented the compilation of the SF by the addition of a point at $2.7 \times 10^{8} \mathrm{~s}-$ by far the largest time lag which is based on timing residuals from 8.5 years of timing observations of PSR B1642-03 at $103 \mathrm{MHz}$ (Shabanova et al. 2001) (Fig. 7, filled circle). We used for this compilation the relation $D_{\mathrm{S}}=(2 \pi f)^{2} D_{\delta t}$ with $D_{\delta t}=2 \sigma_{t}^{2}$ and $\sigma_{t}=1 \mathrm{~ms}$ at $103 \mathrm{MHz}$. $\sigma_{t}$ was taken from the amplitude of timing residuals (Fig. 5 of Shabanova et al. 2001). We estimated the error of $\sigma_{t}$ to be $50 \%$

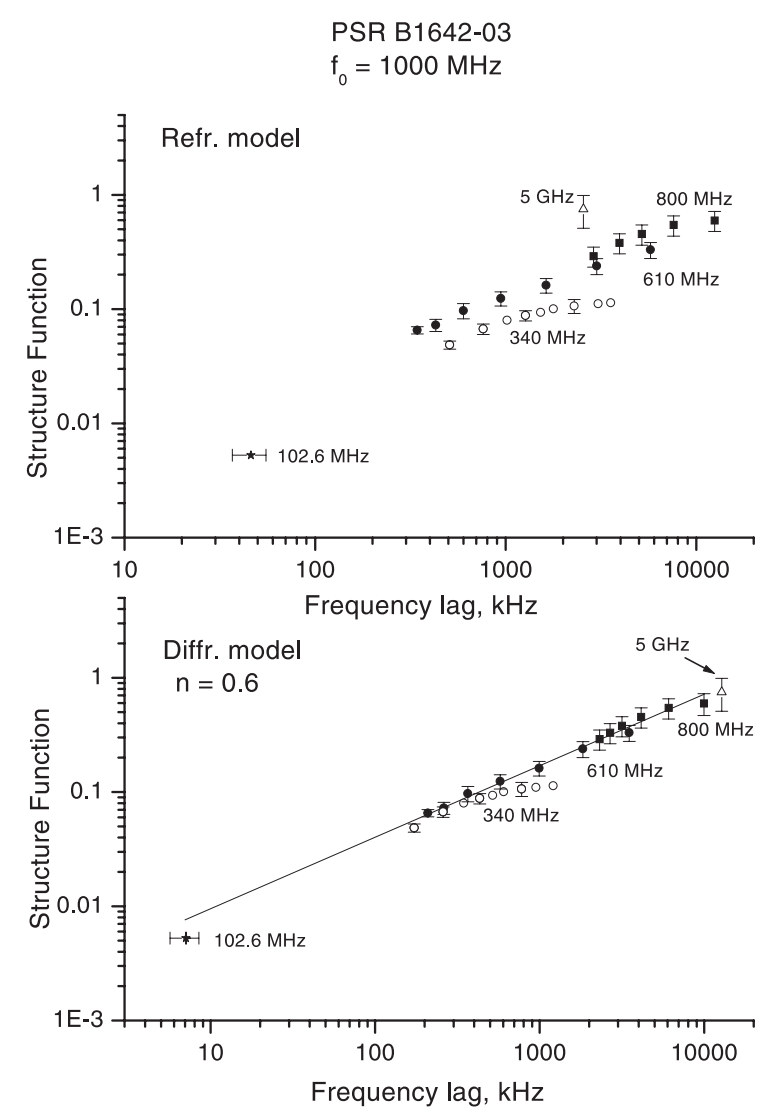

Fig. 8. Frequency structure function of phase fluctuations (in radians ${ }^{2}$ ) reduced to $f_{0}=1 \mathrm{GHz}$ based on data at $102 \mathrm{MHz}, 340 \mathrm{MHz}, 610 \mathrm{MHz}$ and $4.85 \mathrm{GHz}$ (symbols as in Fig. 7) for two models: refractive model (top) and diffractive model (bottom). The solid line corresponds to the best fit to the first data points of the structure function at $610 \mathrm{MHz}$ (diffractive model).

over the 8.5 years interval. The fitted power laws (solid lines) to the composite time SF have a value of $\alpha_{1}=1.35$ up to a scale of about $200 \mathrm{~s}$ and $\alpha_{2}=1.7$ for time lags above $200 \mathrm{~s}$. They match our data well.

The compiled frequency structure function reduced to $f_{0}=$ $1 \mathrm{GHz}$ is shown in Fig. 8. We used two different models for the evaluation: pure diffractive scintillation (shown at the bottom) and scintillation with strong angular refraction (at the top of the figure). At $610 \mathrm{MHz}$ we used the mean structure function (from 3 days of observation) with errors corresponding to $1 \sigma$ deviations. The errors of the SFs at $102.6 \mathrm{MHz}, 340 \mathrm{MHz}$ and $800 \mathrm{MHz}$ presented in Fig. 8 correspond to variations due to refractive scintillation as mentioned earlier. Added to this figure is a point (triangle) which is based on an analysis of the structure function of the $5 \mathrm{GHz}$ data.

The structure function at $5 \mathrm{GHz}$ becomes steeper at the level $D_{\mathrm{S}}=0.03$ (Fig. 3, bottom panel). This steepening is caused by insufficient frequency resolution (our frequency band is $B=$ $500 \mathrm{MHz}$ ) and corresponds to the steepening of the spatial structure function due to averaging of intensity fluctuations in the frequency band (see Lee 1976). We modeled this effect, using our data at $800 \mathrm{MHz}$, since at this frequency we have good frequency resolution. We averaged these data over 20 frequency channels and detected the level where we have a steepening of the calculated $S F(\Delta t)$. Then we used the same level of the frequency structure function to obtain the frequency lag corresponding to this level. This lag was $1000 \mathrm{kHz}$ so the ratio of the real frequency resolution $(20 \times 78 \mathrm{kHz})$ to this lag is: $a=1.56$. This 
coefficient, $a$, will be the same for small time lags of $S F(\Delta t)$ based on data obtained at different frequencies of observation. We conclude therefore that the frequency lag, $\delta f \approx B / 1.56=$ $320 \mathrm{MHz}$ corresponds to the level 0.03 at $5 \mathrm{GHz}$. We converted this level and frequency lag $\delta f$ to $f_{0}=1 \mathrm{GHz}$ and show this point in Fig. 8.

It is obvious that the diffractive model describes our data better: nearly all data points can be fit by one power law with a slope $\beta_{1}=0.6 \pm 0.1$. It might be that the SF steepens at frequency lags higher than $2000 \mathrm{kHz}$, but to decide this $5 \mathrm{GHz}$ observations with better frequency resolution would be needed. The refractive model, by comparison, fails with respect to the $103 \mathrm{MHz}$ and $5 \mathrm{GHz}$ observations, and there are difficulties with the $340 \mathrm{MHz}$ measurements. We are therefore confident that diffractive effects dominate the refractive effects at all frequencies that we used. The slopes of the temporal and frequency structure functions (see Figs. 6 and 8) differ by about a factor of two $\left(\alpha_{1}=1.35 \pm 0.03\right.$ and $\left.\beta_{1}=0.6 \pm 0.1\right)$ as they should for a pure diffractive model. They should be about equal in the refractive model (Shishov et al. 2003), which is ruled out by our data.

\section{Discussion}

We were able to construct the composite time and frequency structure function in the direction to PSR B1642-03 over a wide range of frequencies and decorrelation times based on a comprehensive collection of observations. The measurements show that the interstellar plasma along the line of sight to PSRB1642+03 consists of turbulent irregularities that can be described by a piecewise power law spectrum. The slope of the spectrum is given by $n=\alpha+2$, where $\alpha$ is the slope of the structure function in a log-log diagram, which we found to be either $\alpha=1.7$ for large scales or $\alpha \cong 1.3$ for small scales (time structure function, Fig. 7). This means that the spectrum is close to a Kolmogorov spectrum $(n=3.67)$ for large temporal or spatial scales, similar to the spectrum in the direction to PSR B0329+54 (Shishov et al. 2003). For small scales the spectrum is clearly flatter, with a power law index of $n=3.35 \pm 0.03$ (Fig. 6). The flattening is obvious, both in the time and the frequency structure functions. We did not see such a feature in the direction to PSR B0329+54, and we do not see any influence of angular refraction on the shape of the frequency structure function for PSR B1642-03. For this line of sight we then have

$\theta_{\text {ref }}<\theta_{0}=3.4$ mas for $f=326 \mathrm{MHz}$,

where $\theta_{0}$ is the scattering angle as measured by Gwinn et al. (1993).

The decorrelation time of weak scintillation at $5 \mathrm{GHz}, t_{\text {weak }}=$ $1600 \mathrm{~s}$, can be explained if the thickness $z$ of the scattering layer is

$z=2 \pi f t_{\text {weak }}^{2} \frac{V^{2}}{c} \cong z_{0}\left(\frac{V}{V_{0}}\right)^{2}$,

where $V$ is the transverse velocity of the line of sight relative to the medium, $V_{0}=10 \mathrm{~km} \mathrm{~s}^{-1}$, and $z_{0}=1 \mathrm{pc}$. Here we used $t_{\text {weak }}=r_{\mathrm{Fr}} / V=(z \lambda / 2 \pi)^{0.5} / V$, where $r_{\mathrm{Fr}}$ is the Fresnel scale. For a model where the turbulent medium is located in a thin layer near to the observer, $V$ cannot be larger than $30 \mathrm{~km} \mathrm{~s}^{-1}$ (determined by the motion of the observer) because the component of the Earth's velocity perpendicular to the line of sight in July (the period of the observations at $5 \mathrm{GHz}$ ) is about $10 \mathrm{~km} \mathrm{~s}^{-1}$, and the velocity of the local interstellar medium is about $20 \mathrm{~km} \mathrm{~s}^{-1}$
(Dennet-Thorpe \& de Bruyn 2001). Based on these upper limits of the velocity, Eq. (4) results in $z \leq 10 \mathrm{pc}$. On the other hand, using the relation that determines the characteristic scale of the frequency structure of diffractive scintillation $\Delta f_{\mathrm{d}}$ (see Smirnova et al. 1998) and the value of the scattering angle $\theta_{0}=3.4$ mas (radius of the scattering disk) measured at $326 \mathrm{MHz}$ (Gwinn et al. 1993) one obtains

$z \cong \frac{0.5 c}{\Delta f_{\mathrm{d}} \theta_{0}^{2}} \cong 1 \mathrm{kpc}$.

The numerical coefficient in Eq. (5) corresponds to a power law index $n=3.3$ for a three-dimensional turbulence spectrum. The model of a turbulent medium located in a thin layer near to the observer is, therefore, inconsistent with our data.

If the turbulent medium is uniformly distributed between source and observer one finds from Eq. (4) a distance of $R=$ $z \cong 6.7 \mathrm{kpc}$ to the pulsar, given the measured proper motion of $\Omega=30$ mas/year (Brisken et al. 2003). The pulsar velocity would be about $930 \mathrm{~km} \mathrm{~s}^{-1}$ at such a distance, which is at the extreme end of the pulsar velocity range. The computed value of $R$ is also much larger than the value estimated by Prentice $\&$ ter Haar (1969) which gives $160 \mathrm{pc}$ and the value $2900 \mathrm{pc}$ given by Taylor et al. (1993), so that the model of a uniformly distributed medium is unlikely.

It appears realistic, therefore, to assume that the layer of turbulent medium is located near to the source. If we use $V=\Omega R$ in Eq. (4) and use the known value of $\Omega$ we find $z / R=0.2 R / R_{0}$, where $R_{0}=1 \mathrm{kpc}$ and $R$ is the distance to the pulsar. The same relation was obtained previously (Smirnova et al. 1998). This means that $z$ is either $5 \mathrm{pc}$ for a distance of $160 \mathrm{pc}$ or $1.7 \mathrm{kpc}$ for a distance of $2.9 \mathrm{kpc}$. The corresponding transverse pulsar velocities are $22 \mathrm{~km} \mathrm{~s}^{-1}$ and $400 \mathrm{~km} \mathrm{~s}^{-1}$ for the assumed distances. PSR B1642-03 is a high latitude pulsar $\left(b=26^{\circ}\right)$. It is therefore unlikely that there is strong scattering at $z=1.7 \mathrm{kpc}$. We believe that it is a nearby pulsar, and we will use $R=160 \mathrm{pc}$ and $V=20 \mathrm{~km} \mathrm{~s}^{-1}$ for the conversion of the temporal scale to the spatial scale. The corresponding spatial scale of the structure function is shown in Fig. 7 (top axis). We see that it consists of two components. The SF corresponds to a Kolmogorov spectrum for large scales (from $10^{9}$ to $10^{15} \mathrm{~cm}$ ), which is in accordance with the results obtained for other pulsars (Armstrong et al. 1995; Shishov \& Smirnova 2002). The level of this component is higher than the level of the SF in the direction to PSR B0329+54. This enhancement may be due to a higher level of the electron density.

The small-scale component (scales less than $10^{9} \mathrm{~cm}$ ) corresponds to an unusually flat spectrum of turbulence ( $n$ about 3.3 ). We note that PSR B1642-03 is located in the region of an old supernova remnant - the North Polar Spur (Spoelstra 1972) which means that the flattening of the turbulence spectrum might be caused by the special characteristics of the turbulent medium in this supernova remnant. Such flat spectra were also detected for the strongly anisotropic plasma near to the Sun at distances less than 0.1 astronomical units (Woo \& Armstrong 1979; Armstrong et al. 1990). An enhancement of the solar wind fluctuations at the proton thermal gyroradius was described by Neugebauer (1975) for the more distant regions of the interplanetary plasma. The enhancement of the interstellar plasma turbulence spectrum is located in our case in a region of spatial scales near to $4 \times 10^{8} \mathrm{~cm}$, which is close to the value of the thermal proton gyroradius $\rho_{I}$ as estimated by Chashei \& Shishov (1980). 


\section{Conclusions}

The measurements of turbulence spectra by use of multifrequency observations in the direction to a given pulsar show new features which were undetectable by the usual methods based on ensembles of sources. We evaluated the position and thickness of the turbulent medium causing the scintillation of the pulsar emission. We found unusual features like the enhancement of the ISM turbulence spectrum in a narrow range of scales as detected in the direction to PSR B1642-03 and strong angular refraction as detected in the direction to PSR B0329+54. We hope that the use of this new method will allow us to investigate turbulence spectra for a larger number of pulsars in fine detail, enabling us to obtain information on the physical conditions of the interstellar turbulent plasma.

Acknowledgements. This work was supported by INTAS grant No. 2000-849, NSF grant No. AST 0098685, the Russian Foundation for Basic Research, project codes 03-02-509, 03-02-16522, and the Russian Federal Science and Technology Program in Astronomy. We thank the NRAO, operated by Associated Universities under arrangement with the NSF, for support with the $610 \mathrm{MHz}$ and $800 \mathrm{MHz}$ observations and L. B. Potapova for technical assistance.

\section{References}

Armstrong, J. W., Coles, W. A., Kojima, M., \& Rickett, B. J. 1990, ApJ, 358, 685
Armstrong, J. W., Rickett, B. J., \& Spangler, S. R. 1995, ApJ, 443, 209

Brisken, W. F., Fruchter, A. S., Hernstein, R. M., \& Torsett, S. E. 2003, ApJ, 26, 3090

Chashei, I. V., \& Shishov, V. I. 1980, Soviet Astron. Lett., 6, 301

Cordes, J. M., Weisberg, J. M., \& Boriakoff, V. 1985, ApJ, 288, 221

Dennet-Thorpe, \& de Bryun, A. G. 2001, Astroph. Sp. Sci., 278, 101

Gupta, Y., Rickett, B. J., \& Lyne, A. J. 1994, MNRAS, 269, 1035

Gwinn, C. R., Bartel, N., \& Cordes, J. M. 1993, ApJ, 410, 673

Lee, L. C. 1976, ApJ, 206, 744

Malofeev, V. M., Smirnova, T. V., Soin, A. G., \& Shapovalova, N. V. 1995, Astron. Lett., 21, 619

Neugebauer, J. G. K. 1975, J. Geophys. Res., 80, 998

Ostashev, V. E., \& Shishov, V. I. 1977, Radiophys. Quantum Electron., 20, 581

Prentice, A. J. R., \& ter Haar, D. 1969, MNRAS, 146, 423

Prokhorov, A. M., Bunkin, V. F., Gochelashvily, K. S., \& Shishov, V. I. 1975, Proc. IEEE, 63, 790

Rikett, B. J., Coles, Wm. A., \& Markkanen, J. 2000, ApJ, 533, 304

Roberts, J. A., \& Ables, J. G. 1982, MN, 201, 1119

Shabanova, T. V., Lyne, A. G., \& Urama, J. O. 2001, ApJ, 552, 321

Shishov, V. I. 1974, SvA, 17, 598

Shishov, V. I. 2001, Ap\&SS, 278, 163

Shishov, V. I., \& Smirnova, T. V. 2002, Astron. Reports, 46(9), 731

Shishov, V. I., \& Tokumaru, M. 1996, J. Geomag. Geoelectr., 48, 1461

Shishov, V. I., Smirnova, T. V., Sieber, W., et al. 2003, A\&A, 404, 557

Smirnova, T. V., Shishov, V. I., \& Stinebring, D. 1998, Astron. Reports, 42(6), 766

Spoelstra, T. A. T. 1972, A\&A, 21, 61

Stinebring, D. R., Smirnova, T. V., Hankins, T. H., et al. 2000, ApJ, 539, 300

Taylor, J. H., Manchester, R. N., \& Lyne, A. G. 1993, ApJS, 88, 529

Woo, R., \& Armstrong, J. W. 1979, J. Geophys. Res., 84, 7288 
T. V. Smirnova et al.: Turbulent spectrum to PSR B1642-03, Online Material p 1

\section{Online Material}




\section{Appendix A: Construction of the structure function}

The frequency and time structure functions were evaluated from the corresponding correlation functions in the same way as explained in the paper of Shishov et al. (2003). The relationship between the phase structure function $D_{\mathrm{S}}(t)$ and the correlation function of intensity variations $B_{I}(t)$ is given in the saturated scintillation regime by the equation

$B_{I}(t)=\langle I\rangle^{2} \exp \left[-D_{\mathrm{S}}(t)\right]$.

If $\Delta t_{\mathrm{d}}$ is the characteristic scale of intensity variations we have

$D_{\mathrm{S}}\left(\Delta t_{\mathrm{d}}\right)=1$.

Our simulation has shown that Eq. (A.1) can be reduced, for $t \leq 0.7 \Delta t_{\mathrm{d}}$, to the equation

$D_{\mathrm{S}}(t) \cong \frac{B_{I}(0)-B_{I}(t)}{\langle I\rangle^{2}} \cong \frac{1}{2} \frac{D_{I}(t)}{\langle I\rangle^{2}}$,

where $D_{I}(t)$ is the structure function of the intensity fluctuations. This equation describes the scintillation influence due to small scale inhomogeneities both for weak and for strong scintillation (Shishov et al. 2003). The error of this approximation is $25 \%$ at $t=0.7 \Delta t_{\mathrm{d}}$ and $10 \%$ at $t=0.5 \Delta t_{\mathrm{d}}$. In the strong scintillation regime the exact relation can be used

$D_{\mathrm{S}}(t)=\ln \left[\frac{B_{I}(t)}{\langle I\rangle^{2}}\right]$.

For displacements perpendicular to the line of sight as given by movement of the source with velocity $\boldsymbol{V}_{\perp}-$ assumed to be greater than internal motions within the medium or the observer velocity - the phase structure function is given by

$D_{\mathrm{S}}(t)=\int_{0}^{R} \mathrm{~d} r D\left[\boldsymbol{V}_{\perp} t\left(1-\frac{r}{R}\right)\right]$

where $R$ is the distance from the observer to the source and $r$ is the variable distance parameter ( $r=0$ at the pulsar). $D$ is the gradient of the phase structure function computed in the geometrical optics approximation (Prokhorov et al. 1975). This function may be expressed by (Shishov \& Tokumaru 1996):

$D(\boldsymbol{\rho})=4 \pi\left(\lambda r_{\mathrm{e}}\right)^{2} \int \mathrm{d}^{2} \boldsymbol{q}_{\perp}\left[1-\cos \left(\boldsymbol{q}_{\perp} \boldsymbol{\rho}\right)\right] \Phi_{\mathrm{Ne}}\left(\boldsymbol{q}_{\perp}, q_{\|}\right)$,

where $\rho$ denotes a two-dimensional vector between two points in the plane normal to the line of sight, $\lambda$ is the wavelength, $r_{\mathrm{e}}$ the classical electron radius, $\boldsymbol{q}$ the spatial frequency, $q_{\|}$its component along the line of sight, and $\boldsymbol{q}_{\perp}$ its normal component. $\Phi_{\mathrm{Ne}}$ denotes the squared Fourier spectrum of the electron density fluctuations. It is given in the case of a power law spectrum by

$\Phi_{\mathrm{Ne}}(q)=C_{\mathrm{Ne}}^{2}|\boldsymbol{q}|^{-n}$

where $C_{\mathrm{Ne}}^{2}$ characterizes the level of plasma turbulence. $D(\rho)$ is described by the equation (Smirnova et al. 1998):

$$
\begin{aligned}
& D(\rho)=A(n)\left(\lambda r_{\mathrm{e}}\right)^{2} C_{\mathrm{Ne}}^{2}|\rho|^{\alpha} \\
& \text { with } \\
& A(n)=\frac{2^{4-n} \pi^{3}}{\left[\Gamma^{2}(n / 2) \sin (\pi n / 2)\right]}, \alpha=n-2 .
\end{aligned}
$$

To construct the composite structure function we will convert all our data at different observing frequencies to one given frequency $f_{0}=1000 \mathrm{MHz}$ using the universal conversion factor $\left(\lambda_{0} / \lambda\right)^{2}=\left(f / f_{0}\right)^{2}($ Eqs. (A.6) and (A.8)) resulting in:

$D_{\mathrm{S}}\left(t, f_{0}\right)=\left(\frac{f}{f_{0}}\right)^{2} D_{\mathrm{S}}(t, f)$.

Note that $D_{\mathrm{S}}(t, f)$ is valid for a given time $t$, which must be kept constant when $D_{\mathrm{S}}(t, f)$ is converted to $D_{\mathrm{S}}\left(t, f_{0}\right)$. We can estimate the phase structure function from the frequency structure of intensity variations

$D_{S, 1}(\delta f) \cong \frac{B_{I, \mathrm{dif}}(0)-B_{I, \mathrm{dif}}(\delta f)}{\langle I\rangle^{2}}$

The frequency phase structure function (or the decorrelation of the intensity fluctuations at frequency lag $\delta f$ ) is determined by wave scattering caused by inhomogeneities with spatial scales of the order of $\rho(\delta f)$. For the relation between $\delta f$ and $\rho(\delta f)$ we consider two different models. In one case - the diffractive model the frequency structure is determined by scattering due to inhomogeneities with a scale size of $\rho_{\text {dif }}(\delta f)$. The structure function is then given by (Ostashev \& Shishov 1977; Shishov et al. 2003)

$$
\begin{aligned}
D_{\mathrm{S}, 1}(\delta f) & =D_{\mathrm{S}, 1}\left(\rho_{\mathrm{dif}}(\delta f)\right) \\
& =\int_{0}^{R} \mathrm{~d} r D\left[\left(\frac{r}{R}\right)^{1 / 2}\left(1-\frac{r}{R}\right)^{1 / 2} \rho(\delta f)\right] \\
\rho_{\mathrm{dif}}(\delta f) & \cong\left[\frac{(4-n) \pi}{4}\right]^{1 /(n-2)}\left(\frac{\delta f}{f}\right)^{1 / 2} \rho_{\mathrm{Fr}} \propto f^{-1} \\
\rho_{\mathrm{Fr}} & =\left(\frac{R}{k}\right)^{1 / 2}, \quad k=\frac{2 \pi}{\lambda} .
\end{aligned}
$$

Another type of relation between the frequency correlation function $B_{I}(\delta f)$ of the intensity fluctuations and the spatial phase structure function $D_{\mathrm{S}}(\rho)$ is realized in the case of strong angular refraction. According to Shishov (1974), we may introduce the accumulated angle of refraction $\theta_{\text {ref }}$ at a distance $r$. It is a random function of $r$, which depends only weakly on the coordinates in the plane perpendicular to the line of sight. If the characteristic value of the refraction angle $\theta_{\text {ref }}$ is much bigger than the characteristic value of the diffractive or scattering angle

$\theta_{\text {ref }} \gg \theta_{\text {dif }}$

the frequency structure of the scintillation is determined by the frequency dependence of the displacement of the beam path (Shishov 1974; Shishov et al. 2003).

$D_{\mathrm{S}, 1}(\delta f)=D_{\mathrm{S}, 1}\left(\rho_{\text {ref,eff }}(\delta f)\right)$

$\rho_{\text {ref,eff }}(\delta f) \cong \frac{1}{3} \frac{\delta f}{f} R_{\text {eff }} \theta_{\text {ref,eff }} \propto f^{-3}$.

This equation is correct for $\delta f \leq \Delta f_{\mathrm{d}}(f)$. The difference between $D_{\mathrm{S}}$ and $D_{\mathrm{S}, 1}$ depends on the distribution of the turbulent medium along the line of sight. We can reduce the measured value $D_{\mathrm{S}, 1}(\delta f)$ to a given frequency $f_{0}$ by use of the same universal factor as in Eq. (A.9)

$D_{\mathrm{S}, 1}\left(\delta f, f_{0}\right)=\left(\frac{f}{f_{0}}\right)^{2} D_{\mathrm{S}, 1}(\delta f, f)$.

Here, the value of the frequency scale $\Delta f_{\mathrm{d}}$ must be reduced to the same frequency $f_{0}$. For the diffractive model we have (Shishov et al. 2003)

$\Delta f_{\mathrm{d}}\left(f_{0}\right)=\left(\frac{f_{0}}{f}\right)^{2} \Delta f_{\mathrm{d}}(f)$. 
A different functional dependence of the intensity correlation function on $\Delta f$ applies in the case of strong angular refraction (Shishov et al. 2003)

$\Delta f_{\text {ref }}\left(f_{0}\right)=\left(\frac{f_{0}}{f}\right)^{3} \Delta f_{\text {ref }}(f)$.

The power law indices of the temporal and frequency structure functions of intensity fluctuations are different: $(n-2)$ and $(n-2) / 2$ in case of the diffractive model; the indices are equal and have the value $(n-2)$ for the refractive model.

An estimation of the value of the phase structure function can be obtained from the scintillation index of refractive scintillation $m_{\text {ref }}$ (Smirnova et al. 1998). The refractive scintillation index is determined by the value of the turbulence spectrum in the region of spatial frequencies near $1 / b_{\text {ref }}$, where $b_{\text {ref }}$ is the characteristic spatial scale of refractive scintillation. $m_{\text {ref }}^{2}$ can be expressed as (Shishov \& Smirnova 2002)

$m_{\text {ref }}^{2}=C(n) D_{\mathrm{S}}\left(T_{\mathrm{ref}}\right)\left(\frac{\Delta t_{\mathrm{d}}}{T_{\mathrm{ref}}}\right)^{2}$.

$T_{\text {ref }}$ and $\Delta t_{\mathrm{d}}$ are the characteristic temporal scales of refractive and diffractive scintillation respectively, and $C(n)$ is a numerical coefficient, which depends on the power law index $n$ and on the distribution of the medium along the line of sight. In the case of a statistically homogeneous medium with $4-n \ll 1, A(n)$ is given by

$C(n) \cong 6(4-n), \quad 4-n \ll 1$. 